\title{
ON THE FEKETE-SZEGÖ PROBLEM FOR ALPHA-QUASI-CONVEX FUNCTIONS
}

\author{
H. R. ABDEL-GAWAD
}

\begin{abstract}
Let $Q_{\alpha}(\alpha \geq 0)$ denote the class of normalized analytic alpha-quasi-convex functions $f$, defined in the unit disc, $D=\{z:|z|<1\}$, by the condition

$$
\operatorname{Re}\left[(1-\alpha) \frac{f^{\prime}(z)}{g^{\prime}(z)}+\alpha \frac{\left(z f^{\prime}(z)\right)^{\prime}}{g^{\prime}(z)}\right]>0,
$$

Where $f(z)=z+\sum_{n=2}^{\infty} a_{n} z^{n}$ and where $g(z)=z+\sum_{n=2}^{\infty} b_{n} z^{n}$ is a convex univalent function in $D$. Sharp upper bounds are obtained for $\left|a_{3}-\mu a_{2}^{2}\right|$, when $\mu \geq 0$.
\end{abstract}

\section{Introduction}

Denote by $S$ the class of functions $f$ which are analytic and univalent in $D=\{z$ : $|z|<1\}$ and normalized so that $f(0)=f^{\prime}(0)-1=0$. Thus for $f \in S$ we may write

$$
f(z)=z+\sum_{n=2}^{\infty} a_{n} z^{n}
$$

Let $C$ and $K$ be those subsets of $S$, which are convex and close-to-convex respectively. Then $f \in K$ if, and only if, There exists $g \in C$ such that for $z \in D, \operatorname{Re} \frac{f^{\prime}(z)}{g^{\prime}(z)}>0$. In [3] Noor considered a new subclass $C^{*}$ of univalent function that is $f$ given by (1), belongs to $C^{*}$ if, and only if, $f$ is analytic in $D$ and is such that there exists $g \in C$ satisfying

$$
\operatorname{Re} \frac{\left(z f^{\prime}(z)\right)^{\prime}}{g^{\prime}(z)}>0 \text { For } z \in D .
$$

The function in $C^{*}$ are called quasi-convex and $C \subset C^{*} \subset K \subset S$. It is shown [3] that $f \in C^{*}$ if, and only if, $z f^{\prime} \in K$. Recently the function $f$ called $\alpha$-quasi-convex function has been defined and its properties Studied in [4].

A function $f$, analytic in $D$, is said to be $\alpha$-quasi-convex if, and only if, there exists function $g \in C$ such that, for $\alpha$ real and positive

$$
\operatorname{Re}\left[(1-\alpha) \frac{f^{\prime}(z)}{g^{\prime}(z)}+\alpha \frac{\left(z f^{\prime}(z)\right)^{\prime}}{g^{\prime}(z)}\right]>0 .
$$

Received July 16, 1999.

2000 Mathematics Subject Classification. Primary 30C45.

Key words and phrases. Univalent function, close to convex, quasi-convex, alpha quasi-convex functions. 
The class of such functions will be denoted by $Q_{\alpha}$. A classical theorem of Fekete and Szegö [1] states that for $f \in S$ given by (1)

$$
\left|a_{3}-\mu a_{2}^{2}\right| \leq \begin{cases}3-4 \mu & \text { if } \mu \leq 0 \\ 1+2 e^{-2 \mu(1-\mu)} & \text { if } 0 \leq \mu \leq 1 \\ 4 \mu-3 & \text { if } \mu \geq 1\end{cases}
$$

This inequality is sharp in the sense that for each $\mu$ there exists a function in $S$ such that equality holds. The above inequalities can be improved [2]. In particular for $f \in K$ and given by (1), Keogh and Merkes [2] showed that

$$
\left|a_{3}-\mu a_{2}^{2}\right| \leq \begin{cases}3-4 \mu & \text { if } \quad \mu \leq \frac{1}{3} \\ \frac{1}{3}+\frac{4}{9 \mu} & \text { if } \quad \frac{1}{3} \leq \mu \leq \frac{2}{3} \\ 1 & \text { if } \quad \frac{2}{3} \leq \mu \leq 1 \\ 4 \mu-3 & \text { if } \quad \mu \geq 1\end{cases}
$$

Again, for each $\mu$, there is a function in $K$ such that equality holds. In this paper we solve the Fekete-Szego problem for the class $Q_{\alpha}$ of $\alpha$-quasi-convex functions.

\section{Results}

Theorem. Let $f \in Q_{\alpha}$ and be given by (1), then for $0 \leq \alpha \leq 1$

$$
\left|a_{3}-\mu a_{2}^{2}\right| \leq\left\{\begin{array}{llr}
\frac{3}{1+2 \alpha}-\frac{4 \mu}{(1+\alpha)^{2}} & \text { if } & \mu \leq \frac{(1+\alpha)^{2}}{3(1+2 \alpha)} \\
\frac{1}{3(1+2 \alpha)}+\frac{4(1+\alpha)^{2}}{9(1+2 \alpha)^{2} \mu} & \text { if } & \frac{(1+\alpha)^{2}}{3(1+2 \alpha)} \leq \mu \leq \frac{2(1+\alpha)^{2}}{3(1+2 \alpha)} \\
\frac{1}{1+2 \alpha} & \text { if } & \frac{2(1+\alpha)^{2}}{3(1+2 \alpha)} \leq \mu \leq \frac{(1+\alpha)^{2}}{(1+2 \alpha)} \\
\frac{4 \mu}{(1+\alpha)^{2}}-\frac{3}{(1+2 \alpha)} & \text { if } & \mu \geq \frac{(1+\alpha)^{2}}{(1+2 \alpha)}
\end{array}\right.
$$

This inequality is sharp in the sense that for each $\alpha$ in $[0,1]$ and each $\mu$ in the appropriate range of each part of the theorem, there is an $f$ in $Q_{\alpha}$ such that the " $\leq$ " symbol can be replaced by the "=" symbol in the conclusion of that part of the theorem.

\section{Special cases.}

1) When $\alpha=0$. Then $f \in K$ and we have a result given in [2].

2) Let $a=1$. Then $f \in Q$, quasi-convex function given by (1). Then

$$
\left|a_{3}-\mu a_{2}^{2}\right| \leq\left\{\begin{array}{llr}
1-\mu & \text { if } & \mu \leq \frac{4}{9} \\
\frac{1}{9}+\frac{16}{81 \mu} & \text { if } & \frac{4}{9} \leq \mu \leq \frac{8}{9} \\
\frac{1}{3} & \text { if } & \frac{8}{9} \leq \mu \leq \frac{4}{3} \\
\mu-1 & \text { if } & \mu \geq \frac{4}{3}
\end{array}\right.
$$

Again for each $\mu$, there is a function in $Q$ such that equality holds.

We shall require the following 
Lemma 1 ([5], p.166). Let $h \in p$, i.e., let $h$ be analytic in $D$ and satisfy $\operatorname{Re} h(z)>0$ for $z \in D$, with $h(z)=1+c_{1} z+c_{2} z^{2}+\cdots$ Then

$$
\left|c_{2}-\frac{c_{1}^{2}}{2}\right| \leq 2-\frac{\left|c_{1}\right|^{2}}{2} .
$$

Lemma 2 ([2]). Let $g \in C$, with $g(z)=z+b_{2} z^{2}+b_{3} z^{3}+\cdots$, then for $\mu$ real,

$$
\left|b_{3}-\mu b_{2}^{2}\right| \leq \max (1 / 3,|1-\mu|) .
$$

Proof of Theorem. It follows from (2) that we can write

$$
(1-\alpha) f^{\prime}(z)+\alpha\left(z f^{\prime}(z)\right)^{\prime}=g^{\prime}(z) h(z) .
$$

For $g \in C$ and $h \in p$. Equating coefficients in (4) we obtain

$$
2(1+\alpha) a_{2}=c_{1}+2 b_{2} .
$$

and

$$
3(1+2 \alpha) a_{3}=c_{2}+2 c_{1} b_{2}+3 b_{3} .
$$

so that

$$
\begin{aligned}
a_{3}-\mu a_{2}^{2}= & \frac{1}{(1+2 \alpha)}\left[b_{3}-\frac{(1+2 \alpha) \mu}{(1+\alpha)^{2}} b_{2}^{2}\right]+\frac{1}{3(1+2 \alpha)}\left[c_{2}-\frac{3(1+2 \alpha) \mu}{4(1+\alpha)^{2}} c_{1}^{2}\right] \\
& +\left[\frac{2}{3(1+2 \alpha)}-\frac{\mu}{(1+\alpha)^{2}}\right] c_{1} b_{2} .
\end{aligned}
$$

We consider first the case $\frac{(1+\alpha)^{2}}{3(1+1 \alpha)} \leq \mu \leq \frac{2(1+\alpha)^{2}}{3(1+2 \alpha)}$.

Equation (5) gives

$$
\begin{aligned}
\left|a_{3}-\mu a_{2}^{2}\right| \leq & \frac{1}{(1+2 \alpha)}\left|b_{3}-\frac{(1+2 \alpha) \mu}{(1+\alpha)^{2}} b_{2}^{2}\right|+\frac{1}{3(1+2 \alpha)}\left|c_{2}-\frac{3(1+2 \alpha) u}{4(1+\alpha)^{2}} c_{1}^{2}\right| \\
& +\left[\frac{2}{3(1+2 \alpha)}-\frac{\mu}{(1+\alpha)^{2}}\right]\left|c_{1}\right|\left|b_{2}\right|^{\prime} \\
\leq & \frac{1}{(1+2 \alpha)}\left[1-\frac{(1+2 \alpha)}{(1+\alpha)^{2}} \mu\right]+\frac{1}{3(1+2 \alpha)}\left|c_{2}-\frac{1}{2} c_{1}^{2}\right| \\
& +\frac{1}{3(1+2 \alpha)}\left|\frac{1}{2}-\frac{3(1+2 \alpha) \mu}{4(1+\alpha)^{2}}\right|\left|c_{1}\right|^{2}+\left[\frac{2}{3(1+2 \alpha)}-\frac{\mu}{(1+\alpha)^{2}}\right]\left|c_{1}\right|^{\prime} \\
\leq & \frac{1}{(1+2 \alpha)}\left[1-\frac{(1+2 \alpha)}{(1+\alpha)^{2}} \mu\right]+\frac{1}{3(1+2 \alpha)}\left[2-\frac{\left|c_{1}\right|^{2}}{2}\right] \\
& +\frac{1}{3(1+2 \alpha)}\left|\frac{1}{2}-\frac{3(1+2 \alpha)}{4(1+\alpha)^{2}} \mu\right|\left|c_{1}\right|^{2}+\left[\frac{2}{3(1+2 \alpha)}-\frac{\mu}{(1+\alpha)^{2}}\right]\left|c_{1}\right|^{\prime} \\
= & \Phi(t), \text { say, with } t=\left|c_{1}\right| .
\end{aligned}
$$


Where we have used lemmas 1 and 2 and the fact that $\left|b_{2}\right| \leq 1$. Since the function $\Phi$ attains its maximum at $t_{0}=\frac{2\left[2(1+\alpha)^{2}-3(1+2 \alpha) \mu\right]}{3(1+2 \alpha) \mu}$, it follows that the second inequality in the theorem is established, if $\mu \leq \frac{2(1+\alpha)^{2}}{3(1+2 \alpha)}$. Choosing $b_{2}=b_{3}=1, c_{2}=1, c_{1}=$ $\frac{2\left[2(1+\alpha)^{2}-3(1+2 \alpha) \mu\right]}{3(1+2 \alpha) \mu}$ in $(5)$, shows that the result is sharp if $\mu \geq \frac{(1+\alpha)^{2}}{3(1+2 \alpha)}$, since $\left|c_{1}\right| \leq 2$.

Next, let us that $\mu \leq \frac{(1+\alpha)^{2}}{3(1+2 \alpha)}$. Then

$$
\begin{aligned}
\left|a_{3}-\mu a_{2}^{2}\right| & \leq \frac{3(1+2 \alpha)}{(1+\alpha)^{2}} \mu\left|a_{3}-\frac{(1+\alpha)^{2}}{3(1+2 \alpha)} a_{2}^{2}\right|+\left[1-\frac{3(1+2 \alpha)}{(1+\alpha)^{2}}\right]\left|a_{3}\right|^{\prime} \\
& \leq \frac{3}{(1+2 \alpha)}-\frac{4 \mu}{(1+\alpha)^{2}}
\end{aligned}
$$

Where we have used the result already proved in the case $\mu=\frac{(1+\alpha)^{2}}{3(1+2 \alpha)}$, and the fact that for $f \in Q_{\alpha}$, the inequality $\left|a_{3}\right| \leq \frac{3}{1+2 \alpha}$ holds [4]. Equality is attained by the function $f_{0}$, which is defined by

$$
f_{0}(z)=\frac{z}{\alpha z^{\frac{1}{\alpha}}} \int_{0}^{z} \frac{t^{\frac{1}{\alpha}}}{t(1-t)^{2}} d t=z+\frac{2}{1+\alpha} z^{2}+\frac{3}{1+2 \alpha} z^{3}+\cdots
$$

So that $a_{3}-\mu a_{2}^{2}=\frac{3}{1+2 \alpha}-\frac{4 \mu}{(1+\alpha)^{2}}$ (see e.g. [4]). Suppose next that $\frac{2(1+\alpha)^{2}}{3(1+2 \alpha)} \leq \mu \leq \frac{(1+\alpha)^{2}}{(1+2 \alpha)}$.

We deal first with the case $\mu=\frac{(1+\alpha)^{2}}{(1+2 \alpha)}$. Thus (5) gives

$$
a_{3}-\frac{(1+\alpha)^{2}}{(1+2 \alpha)} a_{2}^{2}=\frac{1}{(1+2 \alpha)}\left(b_{3}-b_{2}^{2}\right)+\frac{1}{3(1+2 \alpha)}\left(c_{2}-\frac{3}{4} c_{1}^{2}\right)-\frac{c_{1} b_{2}}{3(1+2 \alpha)}
$$

and so,

$$
\begin{aligned}
\left|a_{3}-\frac{(1+\alpha)^{2}}{(1+2 \alpha)} a_{2}^{2}\right| & \leq \frac{1}{(1+2 \alpha)}\left|b_{3}-b_{2}^{2}\right|+\frac{1}{3(1+2 \alpha)}\left|c_{2}-\frac{1}{2} c_{1}^{2}\right|+\frac{\left|c_{1}\right|^{2}}{12(1+2 \alpha)}+\frac{\left|c_{1}\right|\left|b_{2}\right|}{3(1+2 \alpha)}, \\
& \leq \frac{1}{3(1+2 \alpha)}\left(1-\left|b_{2}\right|^{2}\right)+\frac{1}{3(1+2 \alpha)}\left(2-\frac{\left|c_{1}\right|^{2}}{2}\right)+\frac{\left|c_{1}\right|^{2}}{12(1+2 \alpha)}+\frac{\left|c_{1}\right|\left|b_{2}\right|}{3(1+2 \alpha)} \\
& =\frac{1}{1+2 \alpha}-\frac{1}{3(1+2 \alpha)}\left[\left|b_{2}\right|-\frac{\left|c_{1}\right|}{2}\right]^{2} \leq \frac{1}{1+2 \alpha}
\end{aligned}
$$

Where we have used lemma 1 , the fact $\left|b_{2}\right| \leq 1$ and the inequality $\left|b_{3}-b_{2}^{2}\right| \leq 1 / 3\left[1-\left|b_{2}\right|^{2}\right]$. See e.g. [6]. Now write,

$$
\begin{aligned}
a_{3}-\mu a_{2}^{2}= & \frac{(1+2 \alpha)}{(1+\alpha)^{2}}\left[3 \mu-\frac{2(1+\alpha)^{2}}{(1+2 \alpha)}\right]\left[a_{3}-\frac{(1+\alpha)^{2}}{(1+2 \alpha)} a_{2}^{2}\right] \\
& +\frac{3(1+2 a)}{(1+\alpha)^{2}}\left[\frac{(1+\alpha)^{2}}{(1+2 \alpha)}-\mu\right]\left[a_{3}-\frac{2(1+\alpha)^{2}}{3(1+2 \alpha)} a_{2}^{2}\right]
\end{aligned}
$$


and the result follows at once on using the theorem already proved for $\mu=\frac{(1+\alpha)^{2}}{(1+2 \alpha)}$ and $\mu=\frac{2(1+\alpha)^{2}}{3(1+2 \alpha)}$. Equality is attained when $f_{1}$ is given by

$$
f_{1}(z)=\frac{1}{\alpha} z^{1-\frac{1}{\alpha}} \int_{0}^{z} t^{\frac{1}{\alpha}-1}\left(1-t^{2}\right)^{-1} d t=z+\frac{1}{1+2 \alpha} z^{3}+\frac{1}{1+4 \alpha} z^{5}+\cdots,
$$

So that $a_{3}-\mu a_{2}^{2}=\frac{1}{1+2 \alpha}$. We finally assume that $\mu \geq \frac{(1+\alpha)^{2}}{(1+2 \alpha)}$. Write

$$
a_{3}-\mu a_{2}^{2}=\left[a_{3}-\frac{(1+\alpha)^{2}}{(1+2 \alpha)} a_{2}^{2}\right]+\left[\frac{(1+\alpha)^{2}}{(1+2 \alpha)}-\mu\right] a_{2}^{2}
$$

and the result follows at once on using again the theorem already proved for $\mu=\frac{(1+\alpha)^{2}}{(1+2 \alpha)}$ and the inequality $\left|a_{2}\right| \leq \frac{2}{1+\alpha}$, which was proved in [4]. Equality is attained by the function $f_{0}$, which is defined above.

\section{References}

[1] M. Fekete and G. Szego, Eine Bermerkung Uber ungerade schlichte Funktionen, J. London Math. Soc., 8(1933), 85-89.

[2] F. R. Keogh and E. P. Merkes, A coefficient inequality for certain classes of analytic functions, Pro. Amer. Math. Soc., 20(1969), 8-12.

[3] K. I. Noor and D. K. Thomas, On quasi-convex univalent functions, Inter. J. Math. and Math. Sci, 3(1980), 255-266.

[4] K. I. Noor and F. M. Alobudi, Alpha-quasi-convex functions, Caribb J. Math., 3(1984), 1-8.

[5] Ch. Pommerenke, Univalent functions, Van denhoeck and Ruprecht, Göttingen, 1975.

[6] S. Y. Trimble, A coefficient inequality for convex univalent functios, Proc. Amer. Math. Soc., 48(1975), 266-267.

Mathematics Department, Faculty of Science, Aswan-Egypt. 